\title{
Imaging Review of Paraneoplastic Neurologic Syndromes
}

\author{
(D) A.A. Madhavan, (D)C.M. Carr, (DP.P. Morris, (D)E.P. Flanagan, (D) A.L. Kotsenas, (D) C.H. Hunt,
} (D) L.J. Eckel, (D)E.P. Lindell, and (D)F.E. Diehn

\begin{abstract}
SUMMARY: Paraneoplastic syndromes are systemic reactions to neoplasms mediated by immunologic or hormonal mechanisms. The most well-recognized paraneoplastic neurologic syndrome, both clinically and on imaging, is limbic encephalitis. However, numerous additional clinically described syndromes affect the brain, spinal cord, and peripheral nerves. Many of these syndromes can have imaging findings that, though less well described, are important in making the correct diagnosis. Moreover, imaging in these syndromes frequently mimics more common pathology, which can be a diagnostic challenge for radiologists. Our goal is to review the imaging findings of paraneoplastic neurologic syndromes, including less well-known entities and atypical presentations of common entities. Specifically, we discuss limbic encephalitis, paraneoplastic cerebellar degeneration, paraneoplastic brain stem encephalitis, cranial neuropathy, myelitis, and polyneuropathy. We also demonstrate common diagnostic pitfalls that can be encountered when imaging these patients.
\end{abstract}

ABBREVIATIONS: ANNA = antineuronal nuclear autoantibody; PNS = paraneoplastic neurologic syndrome; PCD = paraneoplastic cerebellar degeneration

$\mathbf{P}$ araneoplastic syndromes (PNSs) result from systemic reactions to neoplasms, often mediated by immunologic or hormonal mechanisms. PNSs include limbic encephalitis, encephalomyelitis, paraneoplastic cerebellar degeneration (PCD), brain stem encephalitis, polyneuropathy, stiff-person syndrome, retinopathy, myasthenia gravis, Lambert-Eaton myasthenic syndrome, and enteric nervous system dysfunction (Fig 1). These syndromes are often associated with serum or CSF positivity of onconeuronal or neuronal cell surface antibodies. Onconeuronal antibodies are more directly associated with underlying neoplasms and cause neuronal dysfunction by recruitment of cytotoxic T cells. In contrast, neuronal cell surface antibodies are less commonly associated with an underlying neoplasm and mediate pathology by directly binding to neurons. PNSs can occur in the presence or absence of paraneoplastic antibodies and are ultimately a clinical diagnosis. Conversely, antibody-mediated neurologic syndromes can occur in the absence of malignancy, though these are separately classified. ${ }^{1}$

Received May 19, 2020; accepted after revision June 29.

From the Division of Neuroradiology, Department of Radiology (A.A.M., C.M.C., P.P.M., A.L.K., C.H.H., L.J.E., E.P.L., F.E.D.) and the Department of Neurology (E.P.F.), Mayo Clinic, Rochester, Minnesota.

Please address correspondence to Ajay Madhavan, MD, Department of Radiology, Mayo Clinic, 200 First Street SW, Rochester, MN 55905, e-mail:

madhavan.ajay@mayo.edu

- Indicates open access to non-subscribers at www.ajnr.org

Indicates article with supplemental on-line table.

http://dx.doi.org/10.3174/ajnr.A6815
Many PNSs have salient imaging features. Although some of these are well described, particularly those of limbic encephalitis, others are not. Additionally, the spectrum of imaging findings for many PNSs is more variable than what is currently reported in the literature, which can frequently lead to incorrect or delayed diagnoses. Imaging is frequently obtained before the formal diagnosis of a PNS or underlying malignancy. ${ }^{2}$ Therefore, it is important to be aware of imaging features of these syndromes and common pitfalls.

This review illustrates the typical imaging findings of paraneoplastic neurologic syndromes in a pictorial essay format and briefly discusses the differential diagnosis for each syndrome when encountered on imaging (On-line Table). Although prior reviews have comprehensively discussed the clinical features of these syndromes, their associated imaging features have not been widely demonstrated, with the notable exception of limbic encephalitis. The patients presented were ultimately diagnosed with paraneoplastic syndromes based on a combination of clinical, laboratory, and imaging findings. Several of the patients also highlight potential imaging pitfalls that can obfuscate the correct diagnosis.

\section{PARANEOPLASTIC NEUROLOGIC SYNDROMES BY ANATOMIC LOCATION} Brain: Limbic Encephalitis

Limbic encephalitis refers to inflammatory changes involving the limbic system, which includes the hippocampus, amygdala, hypothalamus, and cingulate cortex. Symptoms include mood and 


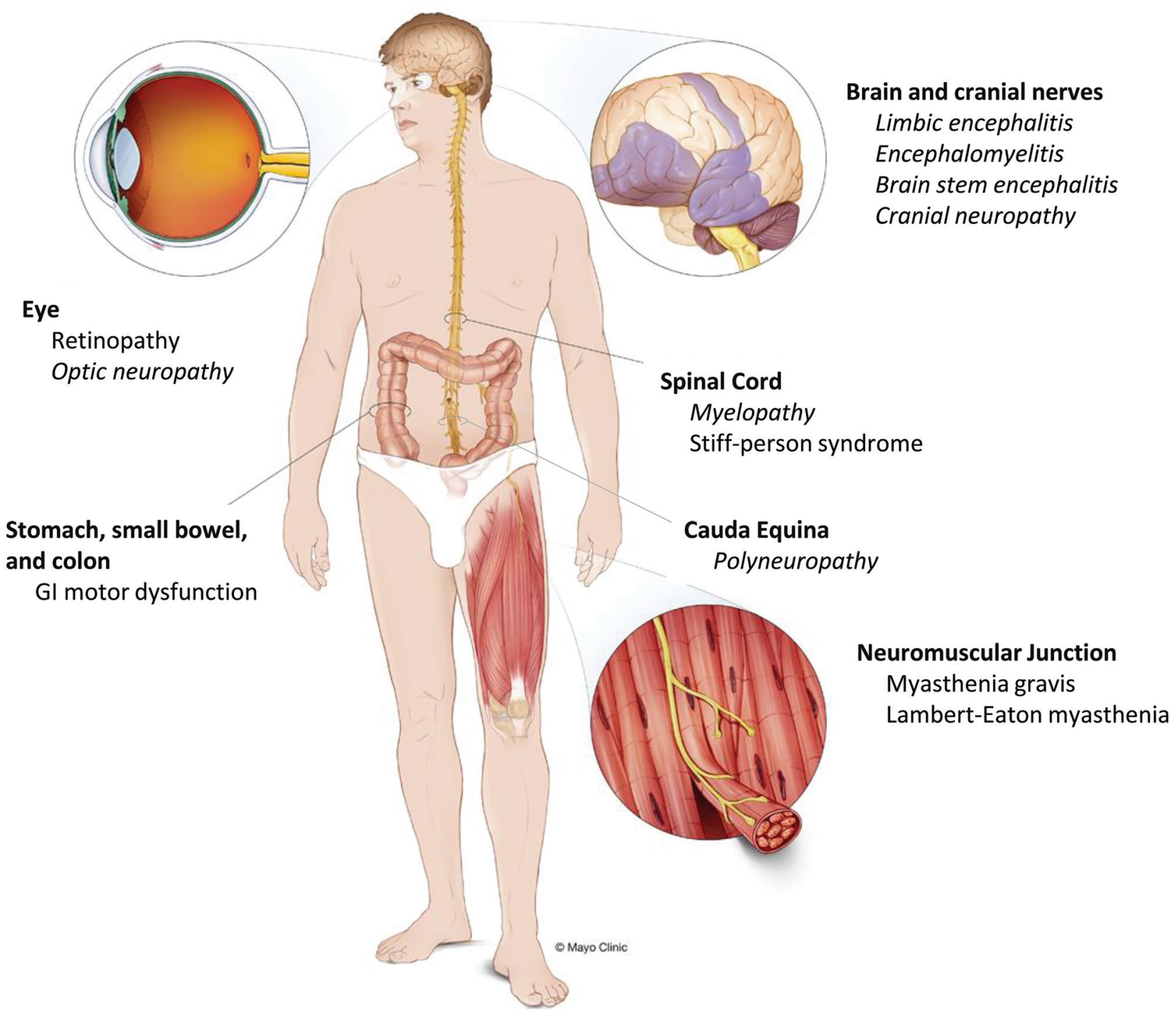

FIG 1. Illustration of the diverse array of paraneoplastic neurologic syndromes affecting a wide variety of anatomic structures. Most of these syndromes (in italics) can have salient imaging findings that can be important in making the correct diagnosis. Used with permission of Mayo Foundation for Medical Education and Research, all rights reserved.

behavioral changes, cognitive dysfunction, memory loss, and seizure activity. Limbic encephalitis may be associated with various onconeuronal paraneoplastic antibodies, including antineuronal nuclear autoantibody type 1(ANNA-1)/anti-Hu, anti-collapsin response mediator protein-5 (CRMP5)/anti-CV2, and anti-Ma2. Although the syndromes associated with these antibodies all characteristically involve the limbic system, some can have extralimbic involvement. For example, anti-Ma2 can involve the brain stem and cerebellum, and anti-CRMP5 can have spinal cord involvement. Common tumor associations for limbic encephalitis in general include small cell lung cancer and breast cancer. Alternatively, limbic encephalitis can be seen in association with nonparaneoplastic neuronal cell surface antibodies, causing an autoimmune encephalitis. These include leucine-rich glioma-inactivated-1 (LGI1) autoantibodies, GAD65 autoantibodies, and anti-contactin-associated proteinlike 2 (CASPR2) antibodies. ${ }^{3,4}$ Patients with autoimmune encephalitis often have typical limbic system involvement, but sometimes different imaging findings such as subcortical T2 hyperintensities are seen. ${ }^{5}$ Imaging findings in paraneoplastic limbic encephalitis are ultimately not reliably distinguishable from nonparaneoplastic autoimmune causes, so it is important to be aware of autoantibodies that are more associated with nonparaneoplastic limbic encephalitis. Additionally, even autoimmune encephalitides associated with a typically nonparaneoplastic antibody have been uncommonly seen with underlying malignancy. Therefore, malignancy screening is nearly always appropriate. ${ }^{6}$

Typical imaging findings of limbic encephalitis include T2 hyperintensity and swelling of the mesial temporal lobes with FDG avidity on PET (Fig $2 A,-B$ ), sometimes with associated enhancement. These findings can also involve other parts of the limbic system. For example, paraneoplastic limbic encephalitis can have striking involvement of the hypothalamus and mammillary bodies with relative sparing of the remaining limbic system (Fig $2 C-F$ ). Sometimes limbic encephalitis can have a masslike appearance that 

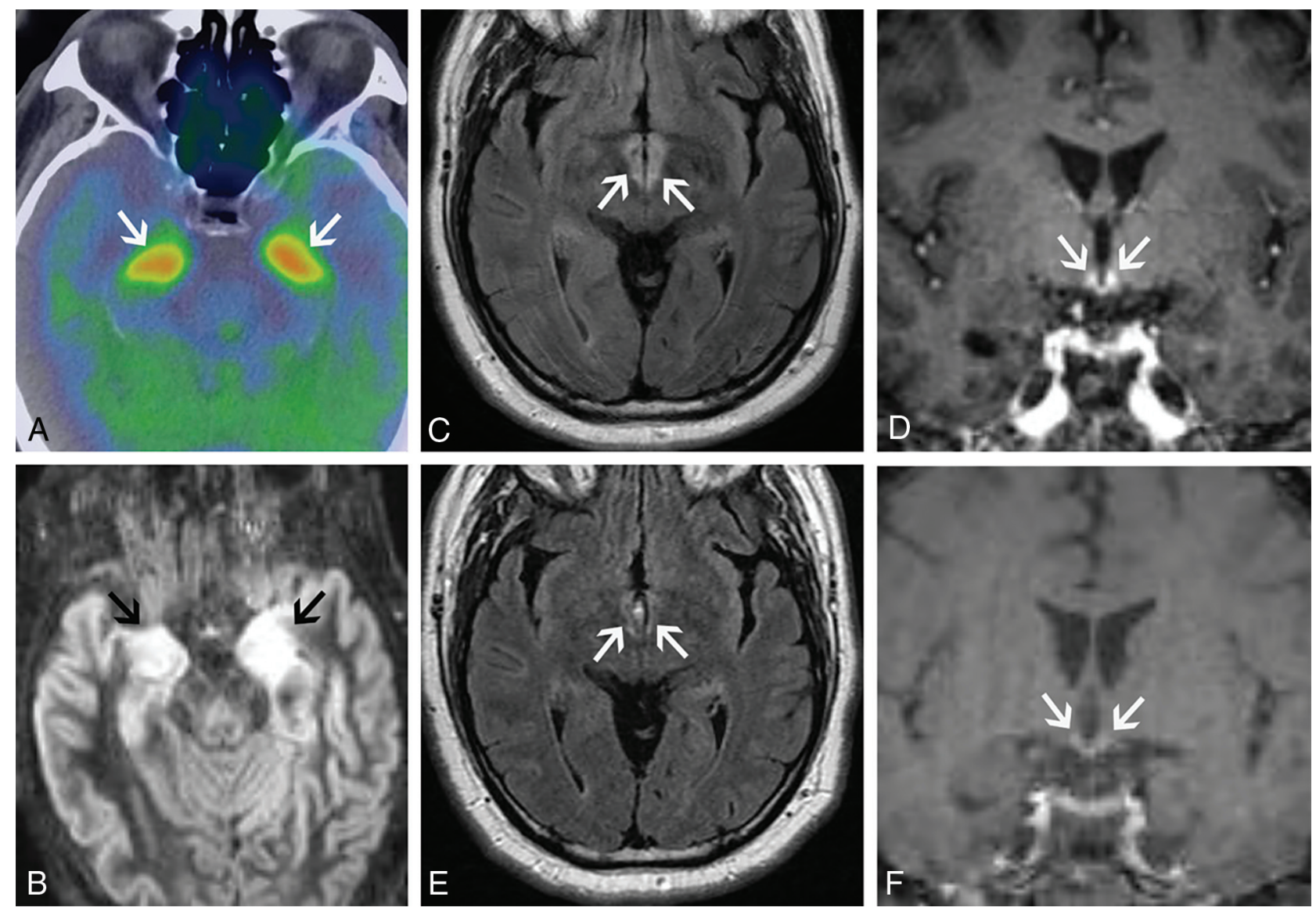

FIG 2. Classic limbic encephalitis ( $A$ and $B$ ). A 75-year-old healthy man presented with abrupt onset of memory loss and staring spells over several days. CSF analysis was positive for LGI1 autoantibodies. FDG-PET/CT axial image (A) shows marked hypermetabolism in the bilateral mesial temporal lobes $(A$, arrows). Axial double inversion recovery MR imaging image $(B)$ shows corresponding increased T2 signal in both mesial temporal lobes $(B$, arrows). Atypical limbic encephalitis (C-F). A 29-year-old man with a history of resected nongerm cell testicular cancer presented months after treatment with worsening memory, diminished executive function, hypogonadism, and hypothyroidism. CSF analysis showed increased total protein but was otherwise normal. Axial FLAIR $(C)$ and coronal T-weighted postcontrast $(D)$ images show abnormal T2 signal in the hypothalamus $(C$, arrows) and enhancement of the mammillary bodies ( $D$, arrows). He was diagnosed with paraneoplastic limbic encephalitis with atypical involvement of the hypothalamus and mammillary bodies. He opted for observation with symptomatic improvement over months. Follow-up MR imaging shows improved mild residual hypothalamic $\mathrm{T} 2$ signal $(E$, arrows) and decreased enhancement of the mammillary bodies ( $F$, arrows).

can be mistaken for tumors such as low- or high-grade gliomas or even lymphoma (Fig $3 A-C$ ). The imaging findings in such cases can dramatically improve with treatment, and FDG hypometabolism may be seen after recovery (Fig $3 D-F)$. Overall, limbic encephalitis has more complex imaging features than previously appreciated and should be considered even in cases that do not involve typical areas such as the hippocampus and amygdala. Atypical patterns of involvement can be seen in nonparaneoplastic limbic encephalitis as well. ${ }^{1,6}$

The primary differential to exclude in limbic encephalitis with mesial temporal lobe involvement is herpes simplex viral encephalitis. Ancillary findings such as cortical petechial hemorrhages, or rarely intracerebral hematomas, can suggest herpes infection. CSF testing and prophylactic antiviral treatment are necessary if infection is a clinical possibility. Other differential considerations include status epilepticus, neurosyphilis, and low- or high-grade glioma. ${ }^{4}$ When limbic encephalitis has hypothalamic involvement, inflammatory conditions such as sarcoidosis or lymphocytic hypophysitis should be considered.
Diagnostic criteria for limbic encephalitis include subacute symptom onset over less than 3 months, limbic system abnormalities on brain MR imaging, EEG showing epileptic or slow-wave activity in the temporal lobes or CSF pleocytosis, and reasonable exclusion of other etiologies. ${ }^{7}$ Thus, the work-up for limbic encephalitis, in addition to a thorough neurologic history and physical examination, typically includes brain MR imaging, paraneoplastic antibody testing, EEG, and oncologic work-up, including FDG-PET/CT. Brain biopsy is rarely needed but can be difficult to avoid when imaging shows a masslike abnormality. After the diagnosis is established, the most important next step is management of any underlying malignancy. ${ }^{8}$ However, immunosuppression and plasma exchange can be helpful, too.

\section{Brain: Paraneoplastic Cerebellar Degeneration}

PCD is clinically characterized by limb and truncal ataxia, nystagmus, and dysarthria. ${ }^{9}$ These symptoms are often progressive over weeks to months. Pathologically, patients have progressive loss of Purkinje cells in the cerebellum. ${ }^{10}$ CSF analysis can show pleocytosis and elevated oligoclonal bands. PCD is associated with a variety of paraneoplastic antibodies, including anti-Yo (Purkinje cell autoantibody type-1 

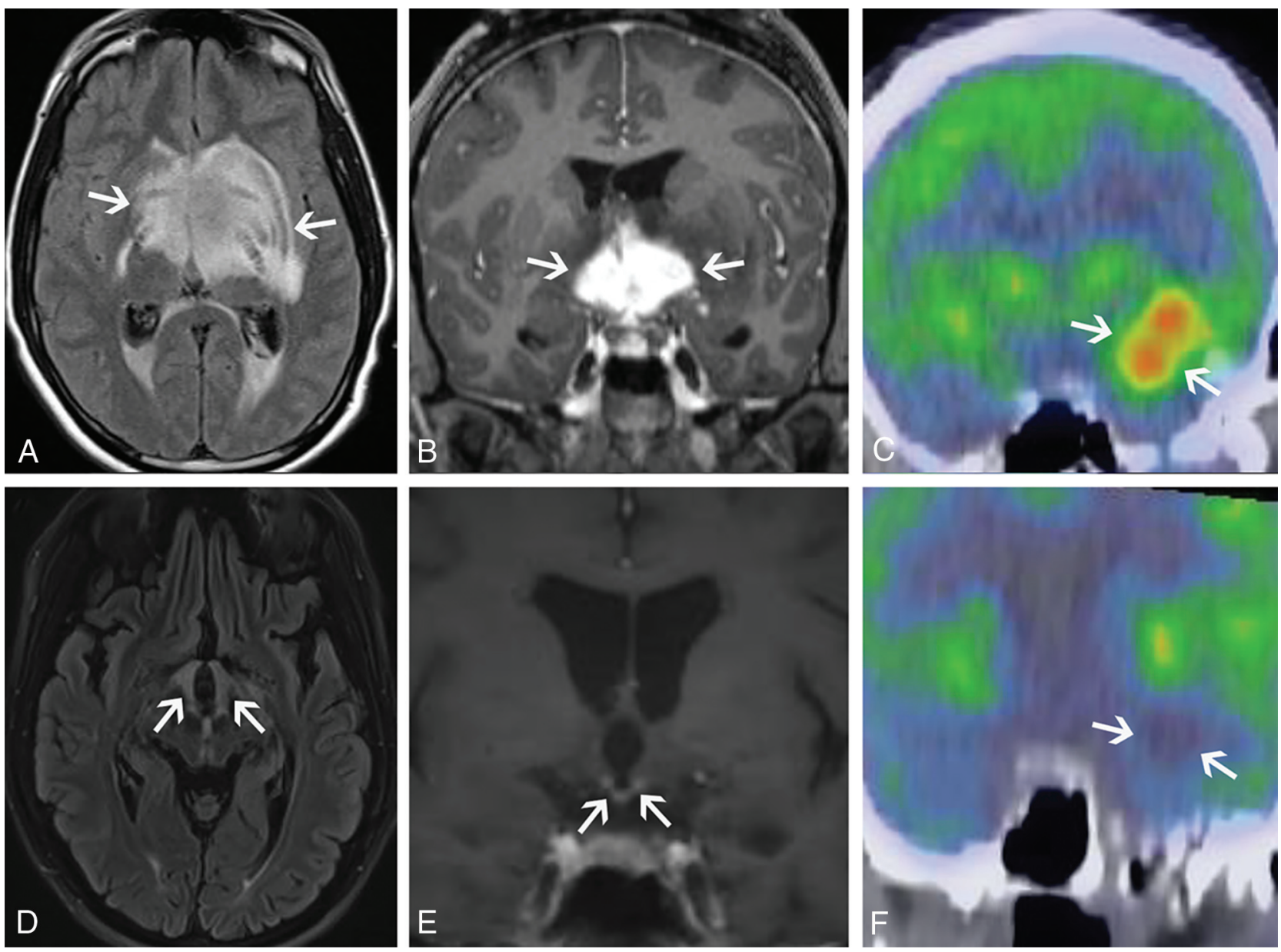

FIG 3. Limbic encephalitis mimicking a tumor. A 31-year-old man presented after being found unconscious while doing repair work on his home He had multiple neurologic symptoms, including impaired thermoregulation, hypersexuality, and depression. Axial FLAIR (A) and coronal T1weighted postgadolinium $(B)$ images show extensive increased $T 2$ signal and masslike enhancement centered in the region of the hypothalamus ( $A$ and $B$, arrows). The findings were initially considered worrisome for a neoplastic process, such as lymphoma, versus an inflammatory lesion, such as lymphocytic hypophysitis. Biopsy of this region showed a nonspecific macrophage-rich demyelinating lesion with no evidence of tumor. He was later found to have anti-Ma2 serum positivity and a mediastinal germ cell tumor. Images of the brain from his staging FDG-PET/CT show asymmetric FDG uptake in the left mesial temporal lobe (C, arrows), separate from the affected regions on his MR imaging. He was treated with chemotherapy and high-dose corticosteroids with gradual improvement in his symptoms. Axial FLAIR $(D)$ and coronal postgadolinium (E) MR imaging obtained 1year later show marked improvement in the prior findings, with mild residual hypothalamic $T 2$ signal $(D$, arrows) and mild enhancement of the tuber cinereum ( $E$, arrows). His subsequent FDG-PET/CT shows decreased FDG avidity in the previously involved left mesial temporal lobe $(F$, arrows). Ultimately, his intracranial findings were consistent with limbic encephalitis rather than malignancy.

[PCA-1]), anti-Ri (ANNA-2), and anti-Purkinje cell autoantibodyTr/DNER (delta/notchlike epidermal growth factor-related receptor). About $60 \%$ of cases have an associated onconeuronal antibody. ${ }^{9}$ Common associated malignancies include ovarian cancer, breast cancer, and Hodgkin lymphoma.

Imaging findings of PCD depend on the phase of the syndrome. In the acute phase, increased T2 signal within the cerebellar hemispheres can be seen (Fig $4 A,-B$ ). At this stage, the differential diagnosis includes infectious or inflammatory cerebellitis. In the chronic phase, T2 hyperintensity improves, and cerebellar FDG-PET hypometabolism, atrophy, or both may be seen (Fig 4C-F). The differential diagnosis for chronic PCD includes alcoholic degeneration, neurodegenerative disorders such as multiple system atrophy (cerebellar subtype), and sequelae from infectious/inflammatory cerebellitis. Careful attention to patient history, such as prior alcohol use disorder or cerebellar infection, is most important in distinguishing these entities.
As with limbic encephalitis, brain MR imaging, paraneoplastic antibody testing, and oncologic work-up are key to diagnosis. Pelvic sonography or MR imaging should also be considered in women because PCD is associated with gynecologic malignancies. ${ }^{11}$ Treatment of any identifiable malignancy is critical, but immunosuppressive agents are also used, ranging from corticosteroids to cyclophosphamide for severe cases. Treatment response is generally poor if antibodies to intracellular antigens are detected (eg, PCA-1/anti-Yo), and most of these patients progress to wheelchair dependence. If an antibody to a cell surface antigen is instead found (eg, metabotropic glutamate receptor [mGluR1]), the prognosis is better.

\section{Brain: Brain Stem Encephalitis}

Brain stem encephalitis, or rhombencephalitis, refers to inflammation predominantly involving the brain stem, though the cerebellar peduncles and hemispheres can be affected too. Paraneoplastic 

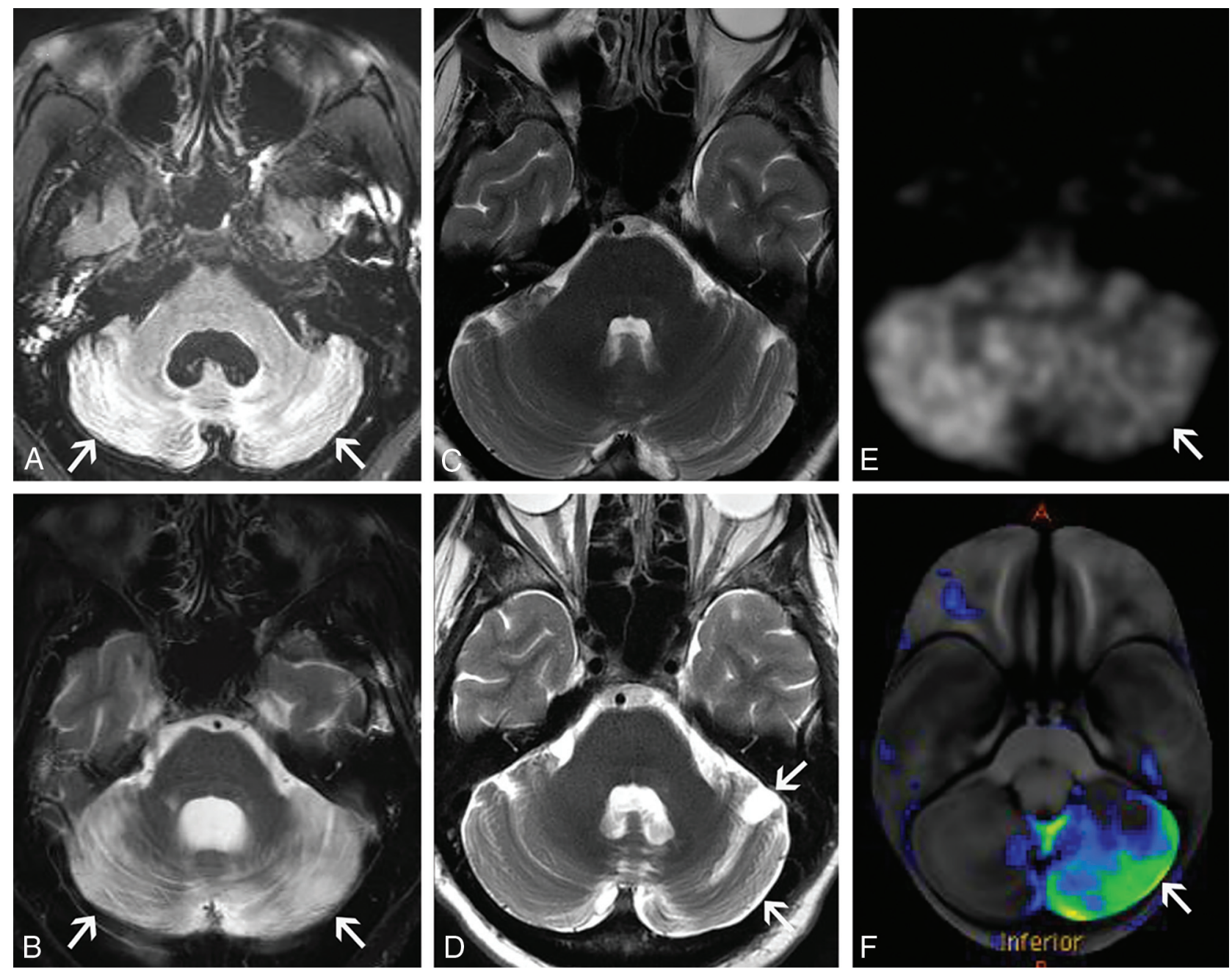

FIG 4. Subacute paraneoplastic cerebellar ataxia ( $A$ and $B)$. A 58-year-old woman presented with headaches, vertigo, and ataxia that had progressed over a period of weeks. CSF analysis showed a high titer of PCA-1 autoantibodies. After additional work-up, she was found to have metastatic breast adenocarcinoma. Axial FLAIR $(A)$ and T2-weighted $(B)$ images of the brain show marked T2 hyperintensity diffusely involving both cerebellar hemispheres ( $A$ and $B$, arrows). No enhancement was seen on postgadolinium images (not shown). The patient was diagnosed with paraneoplastic cerebellar degeneration. She had mild improvement in her neurologic symptoms with high-dose corticosteroids but remained wheelchair dependent. Chronic paraneoplastic cerebellar ataxia $(C-F)$. A 56-year-old woman presented with progressive ataxia over a period of several months. Paraneoplastic serum antibody panel revealed anti-G-AchR (ganglionic acetylcholine receptor) antibodies, and CSF analysis revealed increased protein concentration and high IgG levels. Initial axial T2-weighted image of the brain (C) and subsequent axial T2-weighted image from an examination several months later $(D)$ show interval development of mild left cerebellar atrophy $(D$, arrows). FDG-PET axial source image of the brain $(E)$ and processed surface rendering of the brain compared with age-matched control participants $(F)$ show marked hypometabolism within the left cerebellar hemisphere ( $E$ and $F$, arrows). No cerebral lesion was present to suggest crossed cerebellar diaschisis. Review of prior records noted an FDG-avid thyroid lesion found to be a follicular neoplasm on resection. She was diagnosed with paraneoplastic cerebellar degeneration with chronic atrophic features. Her symptoms have been stable on cyclophosphamide with pulsed corticosteroids.

brain stem encephalitis has a variable clinical presentation that may include ataxia, dysarthria, and ophthalmoplegia. ${ }^{12}$ It is most commonly associated with the anti-Ma2 antibody but has also recently been identified with antibodies to Kelchlike protein $11 .^{13}$ The syndrome is most associated with testicular germ cell tumors such as seminomas. ${ }^{9}$ However, it can also be seen with neuroendocrine and other tumors. ${ }^{14}$

Imaging findings can be normal in these patients. When present, findings vary depending on the phase of illness. Acutely, T2 hyperintensity and/or enhancement of the cerebellum, cerebellar peduncles, and brain stem are seen. Importantly, there can be isolated involvement of the brain stem in the acute phase, which can be mistaken for other entities, including tumor (Fig 5A, -B). As the disease progresses, cerebellar and brain stem atrophy are often seen. Additionally, cruciform T2 hyperintensity in the pons and T2 hyperintensity in the middle cerebellar peduncles can occur with this syndrome secondary to Wallerian degeneration, quite similar to the imaging findings seen in multiple system atrophy (Fig $5 C,-D$ ). Hypertrophic olivary degeneration can also be seen as a result of paraneoplastic brain stem encephalitis (Fig $5 E,-F$ ), presumably caused by disruption of the dentato-rubro-olivary pathway.

Differential considerations for the acute phase are broad, though certain conditions are known to have a predilection for the brain stem. These include Listeria and herpes simplex virus infection as well as inflammatory disorders such as Behçet disease, systemic 

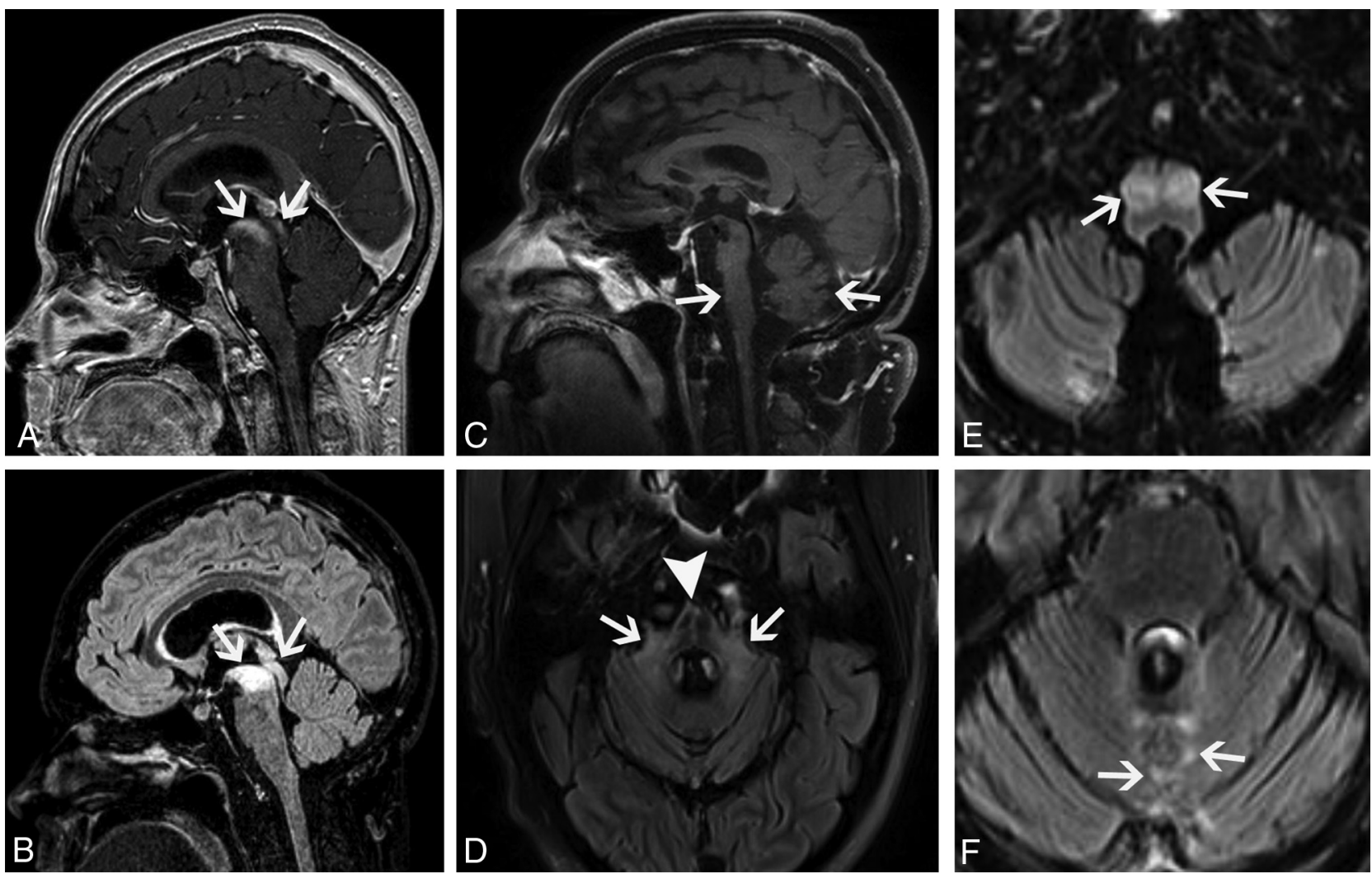

FIG 5. Paraneoplastic brain stem encephalitis mimicking a tumor $(A-D)$. A 29-year-old man presented with tinnitus, hearing loss, diplopia, and imbalance that had progressed over at least 1 year. After extensive clinical work-up, the patient was diagnosed with metastatic seminoma to the mediastinum and noted to have elevated CSF IgG. Serum and CSF were positive for Kelchlike protein 11 autoantibodies. Sagittal T1-weighted postcontrast $(A)$ and sagittal FLAIR $(B)$ images show enhancing, T2 hyperintense lesions in the midbrain and tectum ( $A$ and $B$, arrows), initially believed to be worrisome for metastases. He was treated with chemotherapy, and his symptoms transiently improved but then worsened over the next several years. Sagittal T1-weighted postcontrast $(C)$ and axial FLAIR (D) images from a subsequent MR imaging 4 years later show new marked cerebellar and brain stem atrophy ( $C$, arrows), as well as new T2 signal in the middle cerebellar peduncles ( $D$, arrows) and cruciform $T 2$ signal in the pons ( $D$, arrowhead). He was diagnosed with paraneoplastic brain stem encephalitis rather than multiple system atrophy given the clinical context of the findings. The patient's symptoms mildly improved after treatment with multiple immunosuppressive agents. Brain stem encephalitis associated with hypertrophic olivary degeneration $(E$ and $F$ ). A 45-year-old man presented with progressive aural fullness and hearing loss, initially thought to be caused by otitis media. Over the next 2 years, he had progressive gait ataxia and diplopia. CSF analysis showed antibodies to Kelchlike protein 11. Testicular sonography, performed as part of his oncologic work-up, showed a right testicular mass. Right orchiectomy revealed a germ cell neoplasm. Axial FLAIR images ( $E$ and $F$ ) from his brain MR imaging show enlargement and T2 hyperintensity of both inferior olives $(E$, arrows) consistent with bilateral hypertrophic olivary degeneration. Additionally, there is patchy T2 hyperintensity in the cerebellar vermis ( $F$, arrows). He was diagnosed with paraneoplastic rhombencephalitis with associated hypertrophic olivary degeneration. He has had a partial response to multiple immunotherapies.

lupus erythematosus, Bickerstaff encephalitis, or Miller Fischer syndrome. $^{12,15}$ Demyelinating diseases such as multiple sclerosis, neuromyelitis optica spectrum disorder, and myelin oligodendrocyte glycoprotein antibody-associated disease are additional considerations. In the chronic phase, this syndrome can be mistaken for multiple system atrophy and other neurodegenerative disorders.

In addition to brain MR imaging and usual paraneoplastic antibody screening, screening for the newly discovered KLHL11 autoantibodies should be done when diagnosing these patients. Furthermore, testicular sonography should be done in men given the association with testicular seminoma. ${ }^{11}$ The mainstay of management is treatment of any identifiable malignancy, with immunomodulatory therapy used in refractory cases.

\section{Head: Cranial Neuropathy}

Paraneoplastic cranial neuropathies are rare but have been previously described in several reports. ${ }^{16,17}$ Symptoms depend on the cranial nerves involved. For example, deteriorating balance and sensorineural hearing loss have been observed with paraneoplastic involvement of the eighth cranial nerves. ${ }^{17}$ Symptoms may be more extensive when there is involvement of multiple cranial nerves, as seen in 1 report of paraneoplastic neuropathy of the third, sixth, and seventh cranial nerves in a patient with gallbladder carcinoma. ${ }^{18}$ Anti-Hu/ANNA-1 and the newly described antibodies to Kelchlike protein-11 associated with seminoma are especially associated with paraneoplastic cranial neuropathy.

Possible imaging findings include enhancement and/or enlargement of the affected cranial nerves (Fig 6). The differential 

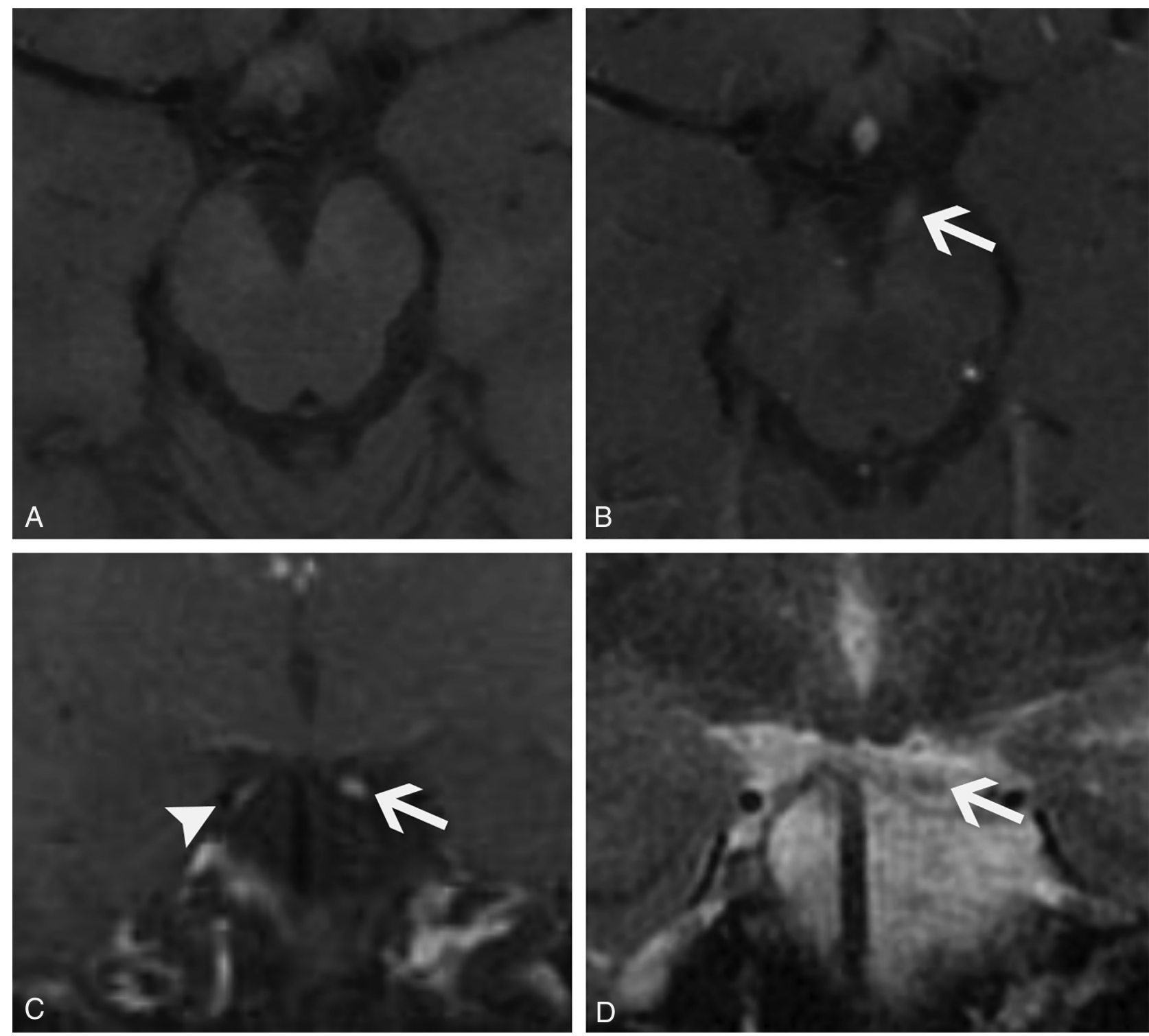

FIG 6. Paraneoplastic cranial neuropathy mimicking leptomeningeal carcinomatosis. A 54-year-old woman recently diagnosed with small cell lung cancer presented with diplopia that slowly progressed over a period of days. Her examination showed left eye ptosis and gaze abnormalities consistent with oculomotor palsy. The primary clinical concern was metastatic leptomeningeal carcinomatosis. Lumbar puncture showed elevated CSF IgG index and IgG synthesis rate with no specific findings for malignancy. No specific paraneoplastic antibodies were detected. Axial T1-weighted pre- $(A)$ and postcontrast $(B)$ images and the coronal postcontrast image $(C)$ from her brain MR imaging show enlargement and enhancement of the left third cranial nerve cisternal segment ( $B$ and $C$, arrows) and mild enhancement of the right third cranial nerve cisternal segment (C, arrowhead). Coronal T1-weighted image (D) confirms mild enlargement of the left third cranial nerve (arrow). Based on the lack of other widespread intracranial disease, non-nodular nerve enhancement, and CSF findings, she was diagnosed with paraneoplastic cranial neuropathy and had rapid symptomatic improvement with methylprednisolone.

diagnosis includes infection, acute inflammatory demyelinating polyneuropathy (Guillain-Barré syndrome), and chronic inflammatory demyelinating polyneuropathy. ${ }^{19,20}$ Entities such as neurosarcoidosis, lymphoma, and carcinomatous meningitis tend to have more nodular enhancement and likely other areas of involvement, whereas chronic inflammatory demyelinating polyneuropathy tends to have mainly nerve root enlargement without enhancement. Notably, leptomeningeal metastases are a frequent clinical concern in patients with cancer who have new cranial neuropathy, so imaging can play an important role in potentially suggesting a paraneoplastic rather than malignant cause (Fig 6).
Diagnosis requires a thorough neurologic work-up, and ophthalmologic consultation is helpful if ophthalmoplegia is identified. Brain MR imaging with thin-section imaging is particularly useful to assess the cranial nerves and patterns of pathologic enhancement. The treatment for paraneoplastic cranial neuropathy is not well studied and mainly involves management of the underlying malignancy. In our experience, patients have improved with short-term corticosteroids.

\section{Spine: Myelitis}

Paraneoplastic myelitis is a rare but likely underreported entity. It may occur in isolation or in conjunction with other involved regions 

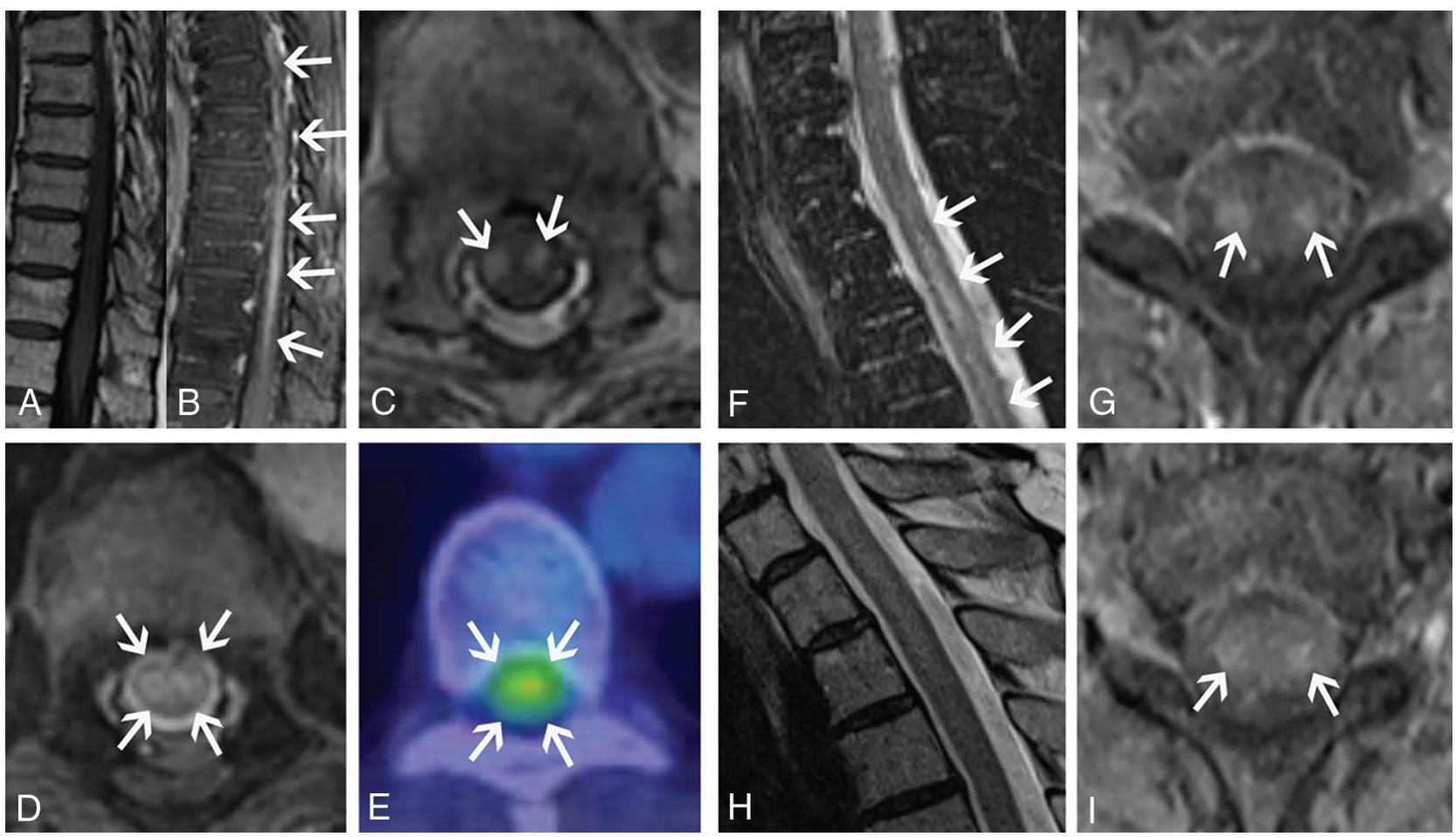

FIG 7. Paraneoplastic myelitis $(A-E)$. A 52-year-old woman recently diagnosed with small cell lung cancer presented with progressive bilateral lower extremity weakness over weeks. Lumbar puncture was remarkable for elevated CSF IgG index and IgG synthesis rate, though no specific paraneoplastic antibody was identified. Sagittal T1-weighted pre- $(A)$ and postcontrast $(B)$ images and an axial postcontrast image (C) from her thoracic spine MR imaging show a long segment of enhancement involving the lateral columns of the thoracic spinal cord. Corresponding axial T2-weighted $(D)$ and FDG-PET/CT $(E)$ images show increased T2 signal ( $D$, arrows) and marked abnormal FDG uptake (E, arrows) in the spinal cord. She was diagnosed with paraneoplastic myelopathy and had transient improvement with methylprednisolone. Paraneoplastic myelitis with treatment response $(F-l)$. A 64-year-old woman recently diagnosed with small cell lung cancer presented with gait difficulties and progressive bilateral lower extremity weakness lasting several months. Serum and CSF were positive for CRMP5 autoantibodies. Sagittal T2-weighted $(F)$ and axial T1-weighted postcontrast $(G)$ images of the cervical cord show increased T2 signal ( $F$, arrows) and enhancement of the lateral columns ( $G$, arrows). She was treated with 6 months of cyclophosphamide and had partial symptomatic improvement. Follow-up sagittal T2-weighted image $(H)$ shows near resolution of abnormal signal. There is mild residual patchy cord enhancement, greatest in the lateral columns (I, arrows).

(eg, paraneoplastic encephalomyelitis). ${ }^{9,21}$ Even in such cases, it differs from limbic encephalitis insofar as it has more generalized cerebral abnormalities not confined to the limbic system. Patients can have an acute myelitis or more insidious progressive myelopathy with combinations of weakness, numbness, and bowel or bladder dysfunction. Other symptoms can include headache, seizures, and agitation, attributable to the associated intracranial involvement. ${ }^{6} \mathrm{~A}$ variety of paraneoplastic antibodies can be seen, but anti-CRMP5/ anti-CV2 and anti-amphiphysin are the most common. Small cell lung and breast cancer are the most frequently associated malignancies. A paraneoplastic aquaporin-4 IgG seropositive neuromyelitis optica spectrum disorder is also recognized. ${ }^{22}$

When confined to the spinal cord, imaging findings include longitudinally extensive T2 hyperintensity and enhancement (Fig 7). Tract-specific abnormalities, particularly along the lateral columns, are characteristic (Fig $7 C,-G$ ). Abnormal FDG uptake can also be present in the involved area of the spinal cord with this syndrome (Fig 7E), which could potentially be mistaken for metastatic tumor involvement. ${ }^{23}$ Differential considerations for this entity include neuromyelitis optica spectrum disorder, neurosarcoidosis, spinal dural AVF, radiation myelitis, and cord infarct. The presence of perimedullary flow voids usually suggests an
AVF, whereas preferential involvement of the anterior horns often suggests cord infarct. Multiple sclerosis would not generally have longitudinally extensive involvement.

In addition to the usual paraneoplastic work-up, MR imaging of the entire spine is particularly helpful in these patients because of the longitudinally extensive nature of spinal cord involvement. Imaging of the brain is also usually indicated to assess for associated encephalitis, which is frequently seen with anti-CRMP5 syndromes. Typical treatment includes management of the underlying malignancy and immunosuppression. Despite treatment, the outcome is overall poor with most patients becoming wheelchair dependent. Although follow-up of patients with paraneoplastic myelitis is not well documented in the literature, clinical improvement and improvement in imaging findings can occur (Fig $7 F-I$ ).

\section{Spine: Polyneuropathy}

Paraneoplastic polyneuropathy is rare but usually manifests clinically as a sensory neuropathy. ${ }^{9}$ This is characterized by pain, paresthesias, and loss of vibratory sensation, often with ANNA-1/anti-Hu or antiCRMP5 antibody positivity. Much less commonly, paraneoplastic syndromes can also have other combinations of motor, sensory, and 

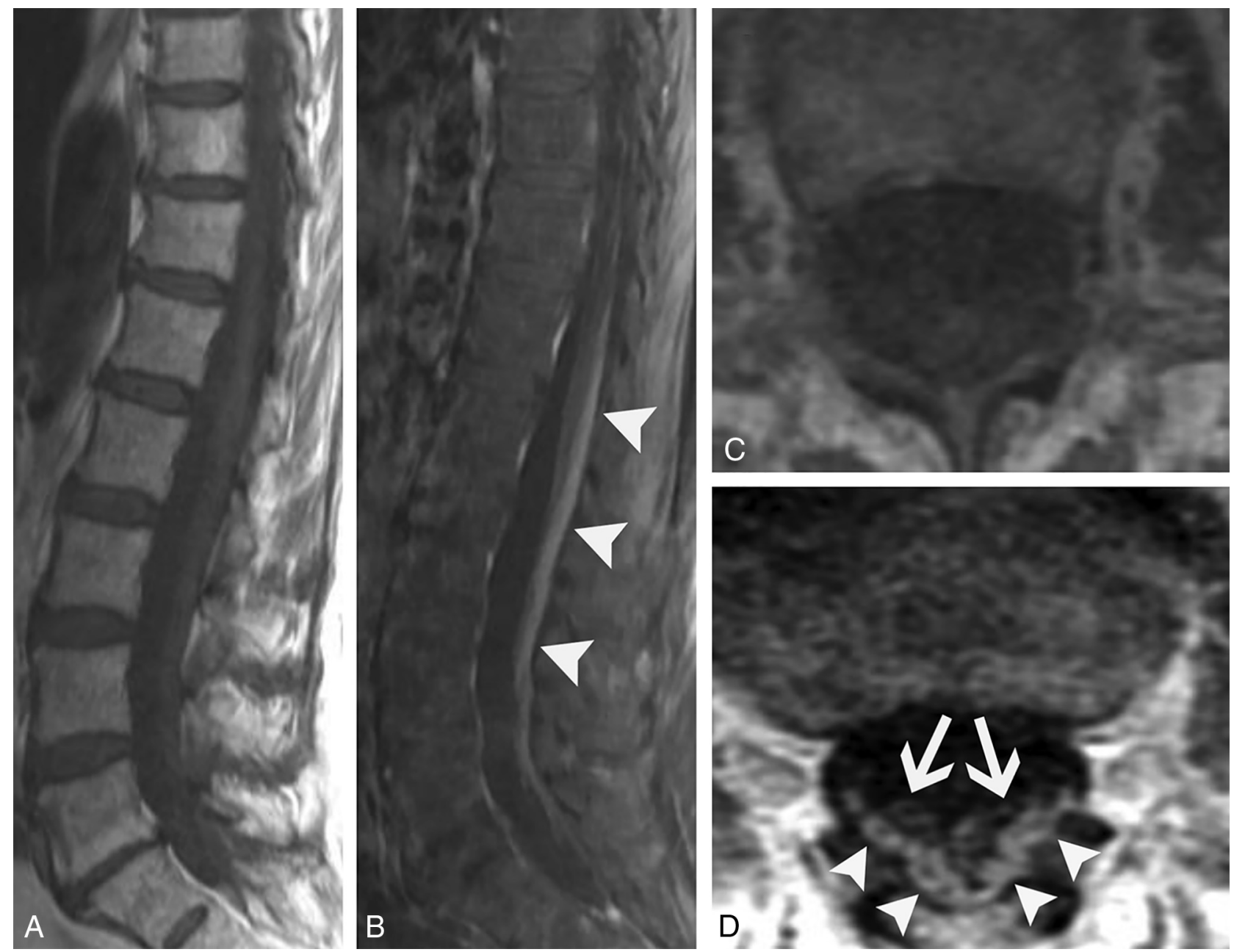

FIG 8. Paraneoplastic polyneuropathy mimicking Guillain-Barré syndrome. A 69-year-old woman presented with rapidly progressive ascending weakness and paresthesias. CSF analysis was remarkable for albuminocytologic dissociation, and the patient was initially diagnosed with GuillainBarré syndrome. Sagittal T1-weighted pre- $(A)$ and postcontrast $(B)$ and axial T1-weighted pre- $(C)$ and postcontrast $(D)$ images from the patient's lumbar spine MR imaging show enhancement of the predominantly dorsal cauda equina nerve roots $(B-D$, arrowheads). There is relative sparing of the ventral nerve roots ( $D$, arrows), somewhat atypical for Guillain-Barré syndrome. The patient's CSF was later found to be ANNA-1/anti-Hu antibody positive, and she was ultimately found to have small cell lung cancer.

autonomic deficits. ${ }^{24}$ As examples, paraneoplastic syndromes associated with Hodgkin lymphoma and prostate cancer causing motor and sensory deficits were recently reported. ${ }^{25-27}$ Autonomic dysfunction was also previously reported from a syndrome related to a carcinoid tumor. ${ }^{28}$

Reports of imaging findings of paraneoplastic polyneuropathy involvement are scarce. To our knowledge, there are only 5 published cases of paraneoplastic polyneuropathy showing cauda equina abnormalities. These have demonstrated variable patterns of enhancement, with 2 patients having ANNA-1 positivity. ${ }^{27,29-32} \mathrm{We}$ have found that enhancement can predominantly involve the dorsal (Fig 8) or ventral nerve roots (Fig 9), and the pattern of enhancement does not always correlate with symptoms. The clinical presentation can frequently mimic Guillain-Barré syndrome with a similar rapidly ascending weakness (Fig 8). Additionally, polyneuropathy may be seen with osteosclerotic multiple myeloma in the setting of POEMS syndrome (polyneuropathy, organomegaly, endocrinopathy, monoclonal plasma proliferative disorder, skin changes). ${ }^{33}$ Although POEMS syndrome is not always described among the classic forms of PNS, many authors consider its peripheral nerve involvement to be paraneoplastic. The presence of sclerotic bone lesions and peripheral nerve root enhancement are the most important imaging features to suggest POEMS syndrome (Fig 10).

Differential diagnoses for paraneoplastic polyneuropathy mainly include Guillain-Barré syndrome and infection. Guillain-Barré syndrome classically, but not always, has ventral predominant nerve enhancement. Chronic inflammatory demyelinating polyneuropathy could be considered but is less likely to show any enhancement and would have more prominent nerve enlargement. Entities such as neurosarcoidosis or leptomeningeal carcinomatosis tend to have more nodular enhancement. Ventral cauda equina enhancement has also recently been described in association with cord infarct. ${ }^{34}$

The diagnostic work-up should include a thorough history to elicit any antecedent infection that may suggest Guillain-Barré 

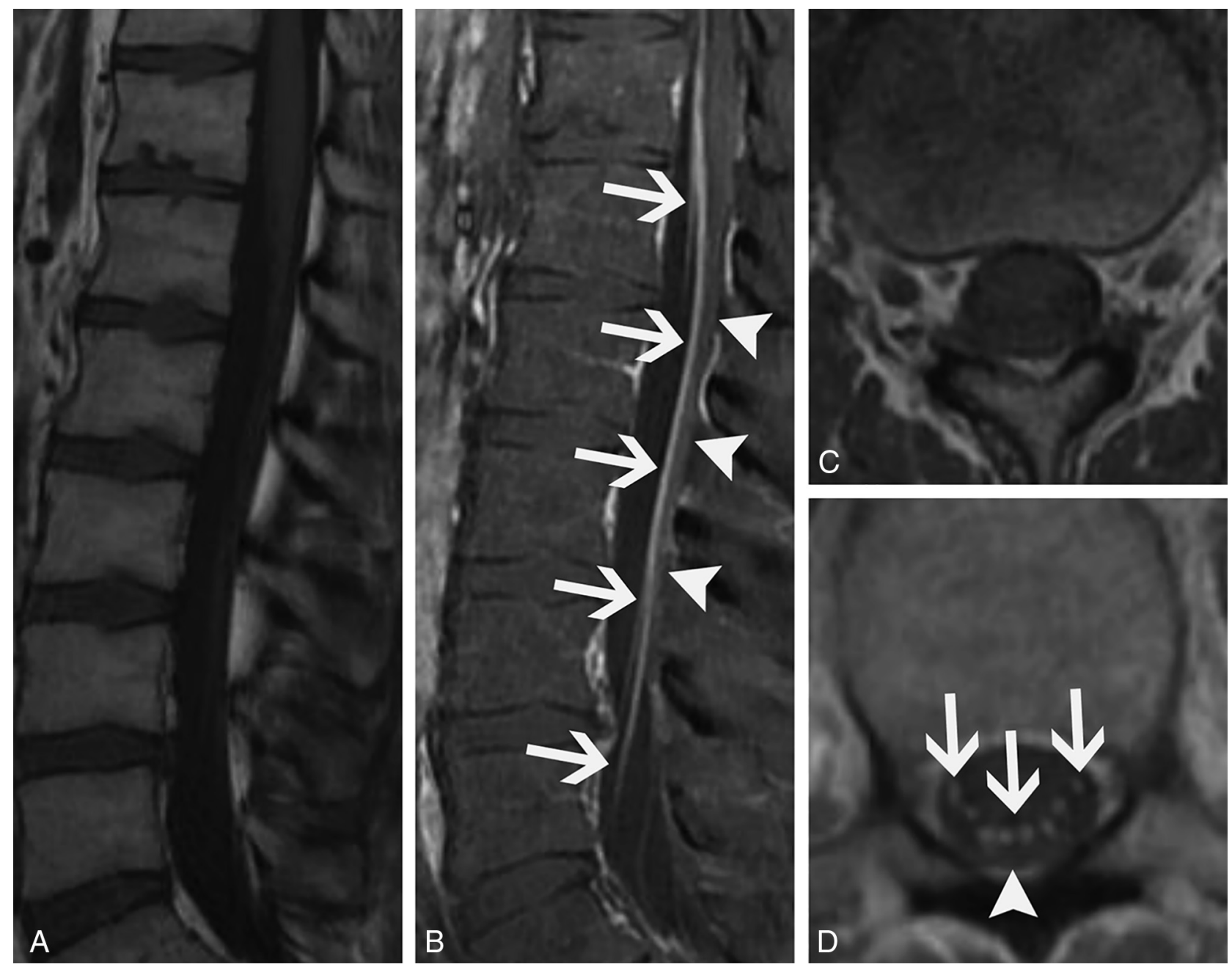

FIG 9. Ventral predominant paraneoplastic polyneuropathy. A 29-year-old man with a history of mediastinal seminoma presented with progressive lower extremity weakness and frequent falls over months. Lumbar puncture showed elevated CSF IgG. Serum and CSF were positive for Kelchlike protein 11 autoantibodies. Sagittal T1-weighted pre- $(A)$ and postcontrast $(B)$ images, as well as corresponding axial T1-weighted pre- $(C)$ and postcontrast $(D)$ images from his lumbar spine MR imaging demonstrate smooth enhancement of the cauda equina nerve roots with a predilection for the ventral roots ( $B$ and $D$, arrows). There is clear sparing of the dorsal roots ( $B$ and $D$, arrowheads). He was diagnosed with paraneoplastic polyneuropathy and had mild symptomatic improvement with numerous immunosuppressive agents.

syndrome, a complete neurologic examination, paraneoplastic antibody testing, electromyography, and lumbar spine MR imaging. Treatment is not well studied, but we have observed symptomatic improvement with corticosteroids and plasma exchange.

\section{CONCLUSIONS}

Paraneoplastic neurologic syndromes can cause a wide range of imaging abnormalities throughout the central and peripheral nervous systems. Prior reports have focused on the imaging findings of limbic encephalitis, but as we have shown, PNSs involving other parts of the nervous system can have characteristic imaging findings. We have described the imaging findings of paraneoplastic limbic encephalitis, cerebellar degeneration, brain stem encephalitis, cranial neuropathy, myelitis, and polyneuropathy. Additionally, we have highlighted the fact that these entities can have variable imaging findings resulting in many potential diagnostic pitfalls. Because imaging features of each entity are sometimes nonspecific, consideration of paraneoplastic syndromes based on imaging findings combined with review of patient history are important. Although serum and CSF paraneoplastic antibody positivity can be helpful, they may be absent, as illustrated in many of our cases. Importantly, many of these syndromes respond to treatment of underlying malignancy or immunotherapy. Therefore, timely diagnosis is likely to significantly impact patient outcomes. Last, it should be noted that PNSs are relatively rare disorders compared with various conditions in the differential diagnosis. The diagnosis of PNS often requires exclusion of these more common entities.

\section{ACKNOWLEDGMENT}

The authors acknowledge the assistance of Desiree J. Lanzino, PT, $\mathrm{PhD}$, in editing the manuscript.

Disclosures: Eoin Flanagan—UNRELATED: Other: Viela Bio, Comments: I am a site principal investigator in a placebo-controlled randomized controlled phase 3 trial on inebilizumab in neuromyelitis optica spectrum disorder. 

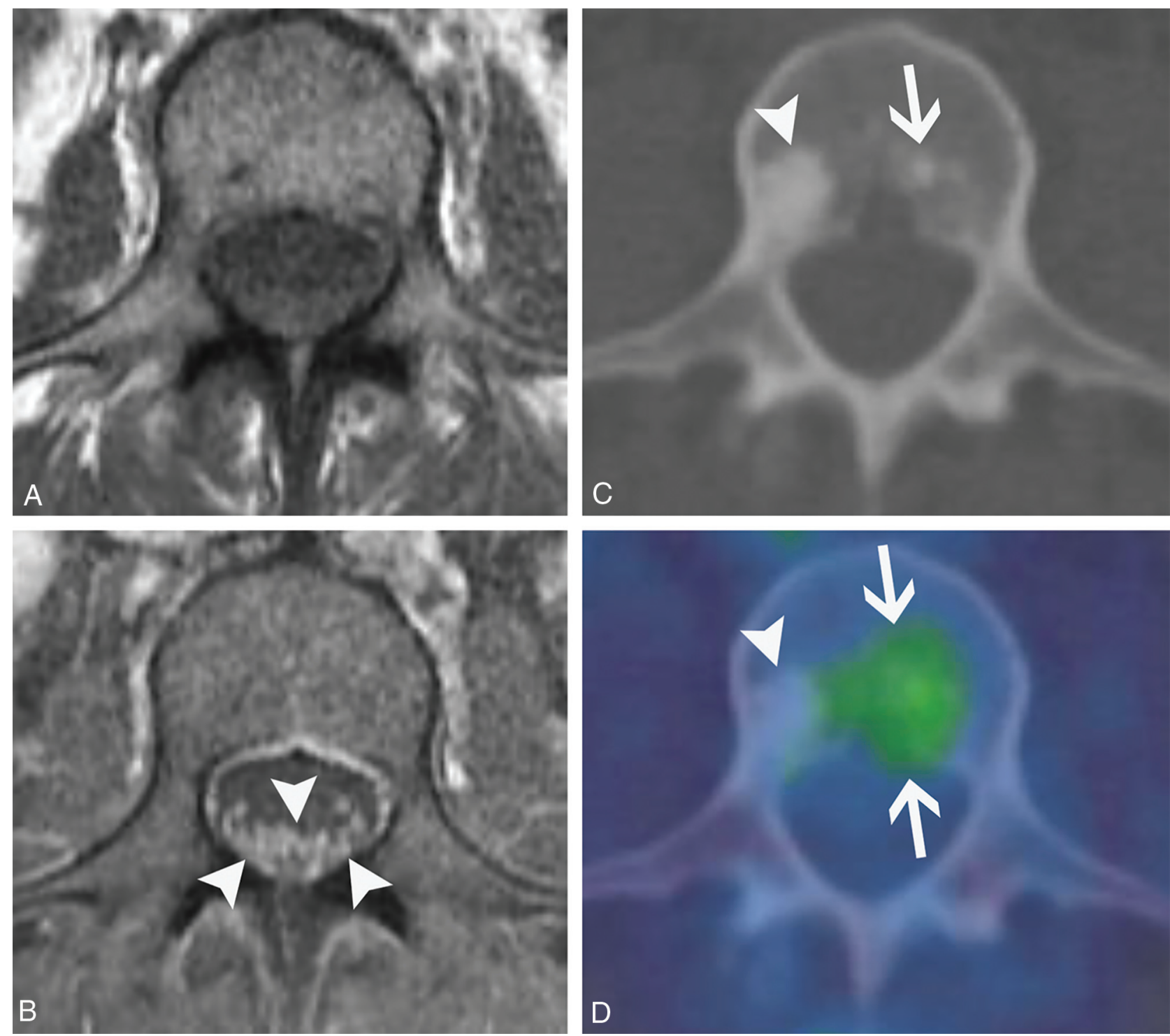

FIG 10. POEMS syndrome. A 50-year-old woman presented with weakness, burning, and tingling in her extremities that had waxed and waned over 4 years. Axial T1-weighted pre- $(A)$ and postcontrast $(B)$ images from her lumbar spine MR imaging show diffuse enlargement and enhancement of the cauda equina nerve roots ( $B$, arrowheads). Axial CT (C) and fused FDG-PET/CT (D) images show a non-FDG-avid lumbar vertebral sclerotic lesion ( $C$ and $D$, arrowheads) and an adjacent mildly FDG-avid sclerotic lesion ( $C$ and $D$, arrows). After additional work-up, she was diagnosed with osteosclerotic multiple myeloma, which is characterized by osteosclerotic lesions with variable FDG avidity. Her MR imaging findings were ultimately attributed to polyneuropathy in the setting of POEMS syndrome.

\section{REFERENCES}

1. Kelley BP, Patel SC, Marin HL, et al. Autoimmune encephalitis: pathophysiology and imaging review of an overlooked diagnosis. AJNR Am J Neuroradiol 2017;38:1070-78 CrossRef Medline

2. Leypoldt F, Wandinger KP. Paraneoplastic neurological syndromes. Clin Exp Immunol 2014;175:336-48 CrossRef Medline

3. Lancaster E, Huijbers MG, Bar V, et al. Investigations of caspr2, an autoantigen of encephalitis and neuromyotonia. Ann Neurol 2011;69:303-11 CrossRef Medline

4. da Rocha AJ, Nunes RH, Maia AC Jr, et al. Recognizing autoimmunemediated encephalitis in the differential diagnosis of limbic disorders. AJNR Am J Neuroradiol 2015;36:2196-205 CrossRef Medline

5. Fredriksen JR, Carr CM, Koeller KK, et al. MRI findings in glutamic acid decarboxylase associated autoimmune epilepsy. Neuroradiology 2018;60:239-45 CrossRef Medline

6. Guerin J, Watson RE, Carr CM, et al. Autoimmune epilepsy: findings on MRI and FDG-PET. Br J Radiology 2019;92:20170869 CrossRef Medline
7. Dutra LA, Abrantes F, Toso FF, et al. Autoimmune encephalitis: a review of diagnosis and treatment. Arq Neuropsiquiatr 2018;76:4149 CrossRef Medline

8. Lancaster $\mathrm{E}$. The diagnosis and treatment of autoimmune encephalitis. J Clin Neurol 2016;12:1-13 CrossRef Medline

9. Hoftberger R, Rosenfeld MR, Dalmau J. Update on neurological paraneoplastic syndromes. Curr Opin Oncol 2015;27:489-95 CrossRef Medline

10. Jarius $S$, Wildemann B. "Medusa head ataxia": the expanding spectrum of Purkinje cell antibodies in autoimmune cerebellar ataxia. Part 3: anti-Yo/CDR2, anti-Nb/AP3B2, PCA-2, anti-Tr/DNER, other antibodies, diagnostic pitfalls, summary and outlook. J Neuroinflammation 2015;12:168 CrossRef Medline

11. Mahta A, Vijayvergia N, Bhavsar TM, et al. Diagnostic approach to a patient with paraneoplastic neurological syndrome. World J Oncol 2012;3:243-46 CrossRef Medline

12. Campos LG, Trindade RA, Faistauer A, et al. Rhombencephalitis: pictorial essay. Radiology Bras 2016;49:329-36 CrossRef Medline 
13. Mandel-Brehm C, Dubey D, Kryzer TJ, et al. Kelch-like protein 11 antibodies in seminoma-associated paraneoplastic encephalitis. $N$ Engl J Med 2019;381:47-54 CrossRef Medline

14. Boch M, Rinke A, Rexin P, et al. Paraneoplastic brainstem encephalitis in a patient with exceptionally long course of a metastasized neuroendocrine rectum neoplasm. BMC Cancer 2014;14:691 CrossRef Medline

15. Jubelt B, Mihai C, Li TM, et al. Rhombencephalitis/brainstem encephalitis. Curr Neurol Neurosci Rep 2011;11:543-52 CrossRef Medline

16. Cheung SSL, Lau GKK, Chan KH, et al. Optic neuritis as the initial clinical presentation of limbic encephalitis: a case report. $J \mathrm{Med}$ Case Rep 2018;12:357 CrossRef Medline

17. Renna R, Plantone D, Batocchi AP. Teaching NeuroImages: a case of hearing loss in a paraneoplastic syndrome associated with antiHu antibody. Neurology 2012;79:e134 CrossRef Medline

18. Kaido M, Yuasa Y, Yamamoto T, et al. A case of possible paraneoplastic neurological syndrome presenting as multiple cranial nerve palsies associated with gallbladder cancer. Rinsho Shinkeigaku 2016;56:617-21 CrossRef Medline

19. Nanda SK, Jayalakshmi S, Ruikar D, et al. Twelfth cranial nerve involvement in Guillain Barre syndrome. J Neurosci Rural Pract 2013;4:338-40 CrossRef Medline

20. Inoue $H$, Tsuboi $Y$, Tsugawa J, et al. Hypertrophic cranial nerve roots in CIDP. Neurology 2004;63:1481 CrossRef Medline

21. Flanagan EP, McKeon A, Lennon VA, et al. Paraneoplastic isolated myelopathy: clinical course and neuroimaging clues. Neurology 2011;76:2089-95 CrossRef Medline

22. Pittock SJ, Lennon VA, de Seze J, et al. Neuromyelitis optica and non organ-specific autoimmunity. Arch Neurol 2008;65:78-83 CrossRef Medline

23. Keegan BM, Flanagan EP. In reply-Sensitivity of [18F]-fluorodeoxyglucose-positron emission tomography in patients with active myelopathy. Mayo Clin Proc 2014;89:859 CrossRef Medline

24. Graus F, Santamaría J, Obach J, et al. Sensory neuropathy as remote effect of cancer. Neurology 1987;37:1266-67 CrossRef Medline
25. Al IO, Koc B, Bayram C, et al. Variant Guillain-Barré syndrome in a patient with Hodgkin lymphoma: AMSAN. Turk Pediatri Ars 2019;53:263-66 CrossRef

26. Choi JK, Kim WJ, Jeon JY. Paraneoplastic sensorimotor polyneuropathy in prostatic adenocarcinoma: a case report. Medicine (Baltimore) 2018;97:e0030 CrossRef Medline

27. Nomiyama K, Uchino A, Yakushiji Y, et al. Diffuse cranial nerve and cauda equina lesions associated with breast cancer. Clin Imaging 2007;31:202-05 CrossRef Medline

28. Veilleux M, Bernier JP, Lamarche JB. Paraneoplastic encephalomyelitis and subacute dysautonomia due to an occult atypical carcinoid tumour of the lung. Can J Neurol Sci 1990;17:324-28 CrossRef Medline

29. Kumar N, Dyck PJ. Hypertrophy of the nerve roots of the cauda equina as a paraneoplastic manifestation of lymphoma. Arch Neurol 2005;62:1776-77 CrossRef Medline

30. Flanagan EP, Sandroni P, Pittock SJ, et al. Paraneoplastic lower motor neuronopathy associated with Hodgkin lymphoma. Muscle Nerve 2012;46:823-27 CrossRef Medline

31. Shibata M, Uchida M, Tsukagoshi S, et al. Anti-Hu antibody-associated paraneoplastic neurological syndrome showing peripheral neuropathy and atypical multifocal brain lesions. Intern Med 2015;54:3057-60 CrossRef Medline

32. Madhavan AA, Guerin JB, Eckel LJ, et al. Dorsal cauda equina nerve root enhancement on magnetic resonance imaging due to ANNA1-associated paraneoplastic polyneuropathy. Neuroradiol J 2020 Apr 15 [Epub ahead of print] CrossRef Medline

33. Paranavitane S, Gooneratne L, Chang T. Polyneuropathy, organomegaly, endocrinopathy, monoclonal band, and skin (POEMS) changes syndrome presenting with a pseudosensory level: a case report. J Med Case Rep 2019;13:384 CrossRef Medline

34. Diehn FE, Hunt CH, Lehman VT, et al. Vertebral body infarct and ventral cauda equina enhancement: two confirmatory findings of acute spinal cord infarct. J Neuroimaging 2015;25:133-35 CrossRef Medline 